\title{
All Exact Solutions of Non-Abelian Vortices from Yang-Mills Instantons
}

\author{
Minoru Eto, ${ }^{1}$ Toshiaki Fujimori, ${ }^{2}$ Muneto Nitta, ${ }^{3}$ and Keisuke Ohashi ${ }^{4}$ \\ 1 Department of Physics, Yamagata University, Yamagata 990-8560, Japan \\ ${ }^{2}$ Department of Physics, National Taiwan University, Taipei 10617, Taiwan \\ 3 Department of Physics, and Research and Education Center for Natural Sciences, \\ Keio University, Hiyoshi 4-1-1, Yokohama, Kanagawa 223-8521, Japan \\ 4 Department of Mathematics and Physics, \\ Graduate School of Science, Osaka City University, \\ 3-3-138 Sugimoto, Sumiyoshi, Osaka, 558-8585, Japan
}

(Dated: July 15, 2018)

\begin{abstract}
We successfully exhaust the complete set of exact solutions of non-Abelian vortices in a quiver gauge theory, that is, the $S[U(N) \times U(N)]$ gauge theory with a bi-fudamental scalar field on a hyperbolic plane with a certain curvature, from $S O(3)$-invariant $S U(2 N)$ Yang-Mills instanton solutions. This work provides, for the first time, exact non-Abelian vortex solutions. We establish the ADHM construction for non-Abelian vortices and identify all the moduli parameters and the complete moduli space.
\end{abstract}




\section{INTRODUCTION}

Since the discovery of non-Abelian vortices [1 3], they have been studied extensively [4 6]. While they are a natural extension of Abelian vortices [7, 8] appearing in conventional superconductors, their analogues also appear in high-density quantum chromodynamics (QCD) showing color superconductivity [9]. In supersymmetric gauge theories, they are Bogomol'nyi-Prasad-Sommerfield (BPS) solitons [10] and are stable not only classically but also perturbatively and non-perturbatively. BPS non-Abelian vortices serve as an elegant tool to demonstrate [11] the coincidence of BPS spectra in four-dimensional gauge theories and two-dimensional sigma models [12]. Non-Abelian vortices also play prominent roles as instantons in non-perturbative dynamics of gauge theories in lower dimensions, similar to the role of Yang-Mills instantons [13] in four dimensions; the non-perturbative partition function has been extensively studied by the vortex counting in $\mathcal{N}=(2,2)$ supersymmetric gauge theories in two dimensions [14], similar to the instanton counting in four dimensions [15].

However, vortex equations are not integrable even in the BPS limit [16], and explicit solutions and the moduli space metric are not available. This is in contrast to the case of the self-dual equations for Yang-Mills instantons, for which the well-known Atiyah-DrinfeldHitchin-Manin (ADHM) construction is available [17]. Thus far, some efforts to obtain the moduli space have been made. The moduli space of non-Abelian vortices was determined without the moduli space metric by solving half of the BPS equations [3]. The moduli space metric was obtained implicitly with a matrix function satisfying a differential equation [18]. The asymptotic metric for well-separated vortices was obtained [19] to study the low-energy scattering [20]. The metrics were also obtained on submanifolds for the coincidence limit [21] and on the symmetry orbits [22]. However, the full moduli space is far beyond our reach because of the non-integrability.

Nevertheless, with changes to the geometry, the situation can become totally different. The BPS Abelian vortex equations on the hyperbolic plane $\mathbb{H}^{2}$ of curvature $-1 / 2$ are integrable [23], and a general formula for the exact moduli space metric has been obtained [24]. The integrability is a consequence of the fact that these vortices are obtained as a dimensional reduction from $S O(3)$-symmetric Yang-Mills instantons on flat space $\mathbb{R}^{4}$ [23]. More generally, the vortex equations on Riemann surfaces $\Sigma$ are integrable, when they are 
obtained from self-dual Yang-Mills equations on $\Sigma \times S^{2}$ [25]. Recently, hyperbolic vortices have been studied extensively [26]. In particular, BPS non-Abelian vortex equations in a quiver gauge theory, i.e., $S[U(N) \times U(N)]$ gauge theory coupled with a bi-fundamental scalar field on a hyperbolic space were obtained from $S O(3)$-symmetric $S U(2 N)$ Yang-Mills instantons in a previous study [27]. However, that study only considered embedding of the Abelian vortex solutions into the diagonal $U(1)^{N}$ subgroup. The same vortices on a flat space were also studied in another work [28]. However, there remain open question on whether these vortices have non-trivial orientational moduli or what the complete set of solutions is.

In this Letter, we construct, for the first time, a complete set of all the exact solutions of non-Abelian vortices in the $S[U(N) \times U(N)]$ gauge theory with a bi-fundamental scalar field on a hyperbolic plane with a certain curvature. We use $S O(3)$-invariant $S U(2 N)$ Yang-Mills instanton solutions. We also establish the ADHM construction for non-Abelian vortices and identify all the moduli parameters and the complete moduli space.

\section{HYPERBOLIC VORTICES FROM $S O(3)$-INVARIANT INSTANTONS}

\section{A. $S[U(N) \times U(N)]$ vortices on a hyperbolic plane}

We consider a hyperbolic plane $\mathbb{H}^{2}$ as the upper half plane with a complex coordinate $z$, with $r \equiv \operatorname{Im} z>0$, endowed with the metric

$$
g_{z \bar{z}}=-\frac{2 R^{2}}{(z-\bar{z})^{2}}=\frac{R^{2}}{2 r^{2}} .
$$

The constant $R$ is related to the scalar curvature $-1 / R^{2}$. See Appendix $\mathrm{A}$.

Let us consider the $U(N) \times U(N)$ gauge theory with gauge fields $A_{z}(z, \bar{z})$ and $\tilde{A}_{z}(z, \bar{z})$, coupled with a single bi-fundamental Higgs field $H(z, \bar{z})$. Note that the overall $U(1)$ gauge group is trivial, and hence, the actual gauge group is $S[U(N) \times U(N)]$. For simplicity, we take the gauge coupling $g$ to be common for all gauge groups. The covariant derivative is $\mathcal{D}_{\bar{z}} H=\left(\partial_{\bar{z}}+i A_{\bar{z}} H-i H \tilde{A}_{\bar{z}}\right)$. In this setup, the action of our model is expressed as

$$
\begin{array}{r}
S=v^{2} \int d^{2} x \sigma \operatorname{tr}\left[\frac{1}{\sigma^{2}}\left|F_{z \bar{z}}\right|^{2}+\frac{1}{\sigma^{2}}\left|\tilde{F}_{z \bar{z}}\right|^{2}+\frac{2}{\sigma}\left|\mathcal{D}_{z} H\right|^{2}+\frac{2}{\sigma}\left|\mathcal{D}_{\bar{z}} H\right|^{2}\right. \\
\left.+\frac{\lambda}{4}\left(H H^{\dagger}-\mathbf{1}_{N}\right)^{2}+\frac{\lambda}{4}\left(H^{\dagger} H-\mathbf{1}_{N}\right)^{2}\right],
\end{array}
$$


where the Higgs field $H$ is rescaled so that its vacuum expectation value $v$ becomes the overall constant of the Lagrangian. The function $\sigma$ is the rescaled hyperbolic metric defined by

$$
\sigma \equiv \frac{g^{2} v^{2}}{2} g_{z \bar{z}}=\frac{g^{2} v^{2} R^{2}}{4 r^{2}}
$$

In this Letter, we consider the critical coupling (the BPS limit) $\frac{\lambda}{4}=1$ and the "integrable" case:

$$
R=\frac{1}{g v} \quad \Longleftrightarrow \quad \sigma=\frac{1}{4 r^{2}} .
$$

The action in Eq. (2) can be rewritten in the following form:

$$
\begin{aligned}
E=v^{2} \int d^{2} x \operatorname{tr}[ & \sigma\left|i \sigma^{-1} F_{z \bar{z}}+H H^{\dagger}-\mathbf{1}_{N}\right|^{2}+\sigma\left|i \sigma^{-1} \tilde{F}_{z \bar{z}}-H^{\dagger} H+\mathbf{1}_{N}\right|^{2}+4\left|\mathcal{D}_{\bar{z}} H\right|^{2} \\
& \left.+2 \mathcal{D}_{\bar{z}}\left(\mathcal{D}_{z} H H^{\dagger}\right)-2 \mathcal{D}_{z}\left(\mathcal{D}_{\bar{z}} H H^{\dagger}\right)+2 i\left(F_{z \bar{z}}-\tilde{F}_{z \bar{z}}\right)\right] .
\end{aligned}
$$

Since the covariant derivative of the scalar field $\mathcal{D}_{\mu} H$ should vanish at the boundary of the hyperbolic plane, the lower bound of the action is given by

$$
S \geq-v^{2} \int_{\mathbb{H}} \operatorname{tr}(F-\tilde{F})
$$

This Bogomol'nyi bound is saturated if the following BPS vortex equations are satisfied

$$
\begin{aligned}
& 0=\mathcal{D}_{\bar{z}} H, \\
& 0=i \sigma^{-1} F_{z \bar{z}}+H H^{\dagger}-\mathbf{1}_{N}, \\
& 0=i \sigma^{-1} \tilde{F}_{z \bar{z}}-H^{\dagger} H+\mathbf{1}_{N} .
\end{aligned}
$$

\section{B. $S U(2 N)$ instanton to $S[U(N) \times U(N)]$ vortices}

Here, we consider the $S O(3)$-rotationally-invariant $S U(2 N)$ Yang-Mills instantons in fourdimensional Euclidean space $\mathbb{R}^{4}$. The $S O(3)$ action on $\mathbb{R}^{4}$ rotates the coordinates $\left(x_{1}, x_{2}, x_{3}\right)$ and leaves the $x^{4}$-axis as fixed, while the $S O(3)$ orbit of a point is $S^{2}$, as shown in Fig. 1. We obtain an upper-half plane $\mathbb{H}^{2}$ by an $S^{2}$-dimensional reduction from $\mathbb{R}^{4}$ with the $S O(3)$ fixed line (the $x^{4}$-axis) removed. Since the classical pure Yang-Mills theory is conformally invariant, the conformal equivalence, $\mathbb{R}^{4}-\mathbb{R} \sim \mathbb{H}^{2} \times S^{2}$, implies that the $S O(3)$-invariant $S U(2)$ instantons are reduced by an $S^{2}$-dimensional reduction to $U(1)$ Abelian-Higgs vortices 


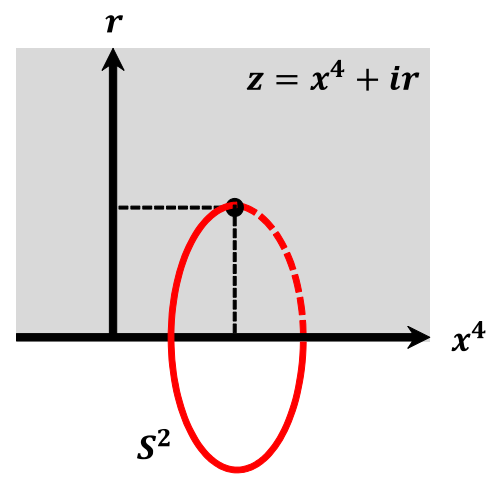

FIG. 1: We consider the $S O(3)$ action on $\left(x^{1}, x^{2}, x^{3}\right)$. The $x^{4}$ axis is a fixed line. The grey region is an upper-half plane or a hyperbolic surface $\mathbb{H}^{2}$.

on a hyperbolic plane $\mathbb{H}^{2}$ with a specific curvature [23]. Here, we extend this relation to the non-Abelian case [27].

First, let us consider the generators of the $S U(2 N)$ gauge group, which are invariant under the diagonal group of the spatial rotation $S O(3)$ and $S U(2) \subset S U(2 N)$ generated by $\mathbf{1}_{N} \otimes \sigma_{i}$. It is convenient to take the following basis for the $S U(2)$-invariant generators

$$
\Lambda=T \otimes P, \quad \tilde{\Lambda}=\tilde{T} \otimes \tilde{P},
$$

where $T$ and $\tilde{T}$ are $N$-by- $N$ Hermitian matrices that can be viewed as the generators of $S[U(N) \times U(N)]$. The 2-by-2 matrices $P$ and $\tilde{P}$ are the projection operators defined by

$$
P \equiv \frac{\mathbf{1}_{2}-\hat{x}_{i} \sigma_{i}}{2}, \quad \tilde{P} \equiv \frac{\mathbf{1}_{2}+\hat{x}_{i} \sigma_{i}}{2}
$$

where $\sigma_{i}(i=1,2,3)$ denote the Pauli matrices. We define the complex coordinate on $\mathbb{H}^{2}$ by $z \equiv x^{4}+i r$ with $r \equiv \sqrt{\left(x^{1}\right)^{2}+\left(x^{2}\right)^{2}+\left(x^{3}\right)^{2}}$, whereas a unit vector for $S^{2}$ is denoted by $\hat{x}_{i} \equiv x_{i} / r$.

The general $S U(2)$-invariant gauge one-form on $\mathbb{R}^{4}$ takes the form

$$
A_{4 d}=A \otimes P+\tilde{A} \otimes \tilde{P}-\frac{1}{2}\left(H-\mathbf{1}_{N}\right) \otimes \omega-\frac{1}{2}\left(H^{\dagger}-\mathbf{1}_{N}\right) \otimes \omega^{\dagger},
$$

where $\omega$ is the $S U(2)$-invariant one-form on $S^{2}$ defined by

$$
\omega \equiv i P \sigma_{i} d \hat{x}_{i}=i \sigma_{i} d \hat{x}_{i} \tilde{P}=\frac{i}{2 r}\left(\delta_{i j}-\hat{x}_{i} \hat{x}_{j}-i \epsilon_{i j k} \hat{x}_{k}\right) \sigma_{j} d x_{i} .
$$


As we will see, the one-forms $A$ and $\tilde{A}$ can be interpreted as the gauge fields on $\mathbb{H}^{2}$. For the gauge field (12), the field strength $F_{4 d}=d A_{4 d}+i A_{4 d} \wedge A_{4 d}$ is given by

$$
\begin{aligned}
F_{4 d}=F & \otimes P+\tilde{F} \otimes \tilde{P}+\frac{1}{2} \mathcal{D} H \otimes \omega+\frac{1}{2} \mathcal{D} H^{\dagger} \otimes \omega^{\dagger} \\
& -\frac{i}{4}\left(H^{\dagger} H-\mathbf{1}_{N}\right) \otimes \omega^{\dagger} \wedge \omega-\frac{i}{4}\left(H H^{\dagger}-\mathbf{1}_{N}\right) \otimes \omega \wedge \omega^{\dagger} .
\end{aligned}
$$

Substituting this field strength into the Yang-Mills action and integrating over $S^{2}$, we find that the $4 \mathrm{~d}$ Yang-Mills action reduces to the action of the $2 \mathrm{~d} U(N) \times U(N)$ gauge theory for the integrable case (4)

$$
\frac{1}{g_{4}^{2}} \int \operatorname{Tr}\left[F_{4 d} \wedge * F_{4 d}\right]=\{\text { Eq. (2) with Eq. (44) }\}, \quad v^{2}=\frac{4 \pi}{g_{4}^{2}},
$$

where we have used the following relations

$$
\begin{aligned}
*(d z \wedge d \bar{z}) \otimes \mathbf{1}_{2} & =r^{2}\left(\omega^{\dagger} \wedge \omega-\omega \wedge \omega^{\dagger}\right) \\
*(d z \wedge \omega) & =-d z \wedge \omega \\
*(d \bar{z} \wedge \omega) & =d \bar{z} \wedge \omega \\
\int_{S^{2}} \frac{i}{2} \operatorname{tr}\left[\omega \wedge \omega^{\dagger}\right] & =4 \pi
\end{aligned}
$$

Similarly, the topological charge of instantons in $4 \mathrm{~d}$ reduces to that of vortices in $2 \mathrm{~d}$

$$
-\frac{1}{g_{4 d}^{2}} \int_{\mathbb{R}^{4}} \operatorname{tr}[F \wedge F]=-v^{2} \int_{\mathbb{H}^{2}}(F-\tilde{F}) .
$$

This implies that the anti-self-dual equation, $F_{4 d}=-* F_{4 d}$, for Yang-Mills instantons reduces to the BPS vortex equations (7), (8), and (9) on a hyperbolic plane $\mathbb{H}^{2}$ for the integrable case (4). Therefore, we can use $S U(2 N)$ instanton solutions to obtain $S[U(N) \times U(N)]$ vortex solutions.

\section{C. $S U(2 N)$ instantons from the ADHM construction}

In order to construct $S O(3)$-invariant instanton solutions, we use the ADHM construction [17]. Let $B_{1}$ and $B_{2}$ be $k \times k$ complex matrices and $I$ and $J$ be $k \times 2 N$ and $2 N \times k$ complex matrices, respectively. Then, "the zero-dimensional Dirac operator" is defined by

$$
\nabla^{\dagger}=\left(\begin{array}{c|cc}
I & z_{2}-B_{2} & z_{1}-B_{1} \\
J^{\dagger} & -\left(\bar{z}_{1}-B_{1}^{\dagger}\right) & \bar{z}_{2}-B_{2}^{\dagger}
\end{array}\right),
$$


where we have defined $z_{1} \equiv i x_{1}+x_{2}, z_{2} \equiv x_{4}+i x_{3}$. Now, let us consider the following $S O(3)$ action on the ADHM data $\left(B_{i}, I, J\right)$

$$
\nabla^{\dagger} \rightarrow g \nabla^{\dagger} h, \quad g=\mathbf{1}_{k} \otimes U^{\dagger}, \quad h=\left(\begin{array}{l|l}
\mathbf{1}_{N} \otimes U & \\
\hline & \mathbf{1}_{k} \otimes U
\end{array}\right),
$$

where $U$ is an arbitrary $S U(2)$ matrix. The most general ADHM data $\left(B_{i}, I, J\right)$ which are invariant under the $S O(3)$ transformation take the following forms (see Appendix $\mathrm{B}$ )

$$
B_{1}=B_{1}^{\dagger}=0, \quad B_{2}=B_{2}^{\dagger}=T, \quad\left(\begin{array}{c}
I \\
J^{\dagger}
\end{array}\right)=\left(\begin{array}{c|c}
\psi & 0 \\
\hline 0 & \psi
\end{array}\right)=\psi \otimes \mathbf{1}_{2},
$$

where $T$ is an arbitrary $k$-by- $k$ Hermitian matrix and $\psi$ is an arbitrary $k$-by- $N$ matrix. We can show that the $S O(3)$-invariant ADHM data automatically satisfy the following ADHM equations.

$$
0=\left[B_{1}, B_{1}^{\dagger}\right]+\left[B_{2}, B_{2}^{\dagger}\right]+I I^{\dagger}-J^{\dagger} J, \quad 0=\left[B_{1}, B_{2}\right]+I J
$$

More generally, the ADHM equations are satisfied if $B_{1}$ and $B_{2}$ are diagonal and $\left(I, J^{\dagger}\right)$ take the form (23). In such a case, the operator $\nabla^{\dagger}$ is given by

$$
\nabla^{\dagger}=\left(\psi \otimes \mathbf{1}_{2}\left(x^{\mu}-T^{\mu}\right) \otimes \bar{e}_{\mu}\right)
$$

where $e_{\mu}=\left(-i \sigma_{i}, \mathbf{1}_{2}\right)$ and $\bar{e}_{\mu}=\left(i \sigma_{i}, \mathbf{1}_{2}\right)$, and we have taken $B_{1}=i T_{1}+T_{2}$ and $B_{2}=i T_{3}+T_{4}$ with $k \times k$ mutually commuting Hermitian matrices $T_{\mu}$. For notational simplicity, first, we deal with the case of the mutually commuting matrices $T_{\mu}$ and then return to the $S O(3)$ invariant case by setting $T_{4}=T$ and $T_{i}=0(i=1,2,3)$.

For the operator $\nabla^{\dagger}$ of the form (25), the zero modes $V$, which are a $(2 N+2 k) \times 2 N$ complex matrix satisfying the equation

$$
\nabla^{\dagger} V=0
$$

are found to be

$$
V=\left(\begin{array}{c}
\mathbf{1}_{N} \otimes \mathbf{1}_{2} \\
-\left(x^{\nu}-T^{\nu}\right)\left[\left(x^{\mu}-T^{\mu}\right)^{2}\right]^{-1} \psi \otimes e_{\nu}
\end{array}\right)\left(S^{\dagger-1} \otimes \mathbf{1}_{2}\right) .
$$

Here, $S$ is an $N$-by- $N$ matrix determined from the orthogonality condition

$$
V^{\dagger} V=\mathbf{1}_{N}
$$


or equivalently

$$
S S^{\dagger}=\mathbf{1}_{N}+\psi^{\dagger}\left[\left(x^{\mu}-T^{\mu}\right)^{2}\right]^{-1} \psi
$$

where we have used the identity $\bar{e}_{\mu} e_{\nu}+\bar{e}_{\nu} e_{\mu}=2 \delta_{\mu \nu} \mathbf{1}_{2}$. From the matrix $V$, the instanton solutions can be explicitly given by

$$
A_{4 d}=-i V^{\dagger} d V=-\frac{i}{2} S^{-1} \partial^{\mu} S \otimes\left(\delta_{\mu \nu} \mathbf{1}_{2}-i \eta_{\mu \nu}^{(+)}\right) d x^{\nu}+\text { (h.c.) }
$$

where $\eta_{\mu \nu}^{(+)}$is the self-dual 't Hooft tensor defined by $\eta_{\mu \nu}^{(+)}=\frac{1}{2 i}\left(\bar{e}_{\mu} e_{\nu}-\bar{e}_{\nu} e_{\mu}\right)$. This solution can be viewed as a generalization of the 't Hooft's multi-instanton configuration for the $S U(2)$ gauge group.

For our purpose, we impose the $S O(3)$ invariance by setting $T_{4}=T$ and $T_{i}=0 \quad(i=$ 1,2,3). In this case, Eq. (29) indicates that matrix $S$ is independent of the coordinates of $S^{2}$. Thus, the solutions become

$$
\left.A_{4 d}=i\left[-S^{-1} \partial_{\bar{z}} S \otimes P+\partial_{\bar{z}} S^{\dagger} S^{\dagger-1} \otimes \tilde{P}\right] d \bar{z}-i r\left(S^{-1} \partial_{z} S+\partial_{z} S^{\dagger} S^{\dagger-1}\right) \otimes \omega+\text { (h.c. }\right),
$$

where we have used

$$
\begin{aligned}
\left(\delta_{\mu \nu} \mathbf{1}_{2}-i \eta_{\mu \nu}^{(+)}\right) d x^{\nu} \partial_{\mu} & =\left(\bar{e}_{\mu} d x^{\mu}\right) e^{\nu} \partial_{\nu} \\
& =2(d z P+r \omega) \partial_{z}+2\left(d \bar{z} \tilde{P}-r \omega^{\dagger}\right) \partial_{\bar{z}}+\text { derivatives on } S^{2}
\end{aligned}
$$

\section{ALL EXACT $S[U(N) \times U(N)]$ VORTEX SOLUTIONS}

\section{A. Exact solutions}

Comparing Eq. (12) with Eq. (31), we can obtain the vortex solutions $A_{z}$ and $H$. Let $T$ be a $k \times k$ Hermitian matrix and $\psi$ be a $k \times N$ complex matrix, made of the respective moduli parameters. The general form of the vortex solution is

$$
A_{\alpha}=-i W^{\dagger} \partial_{\alpha} W, \quad \tilde{A}_{\alpha}=-i \tilde{W}^{\dagger} \partial_{\alpha} \tilde{W}, \quad H=W^{\dagger} \tilde{W}, \quad(\alpha=z, \bar{z}),
$$

where $W$ and $\tilde{W}$ are $(N+k) \times k$ matrices, given by

$$
W \equiv\left(\begin{array}{c}
\mathbf{1}_{N} \\
(\bar{z}-T)^{-1} \psi
\end{array}\right) S^{\dagger-1}, \quad \tilde{W} \equiv\left(\begin{array}{c}
\mathbf{1}_{N} \\
(z-T)^{-1} \psi
\end{array}\right) S^{\dagger-1}
$$


with $S(z, \bar{z})$ satisfying

$$
S S^{\dagger}=\mathbf{1}_{N}+\psi^{\dagger}[(z-T)(\bar{z}-T)]^{-1} \psi
$$

Here, $S$ is the same matrix as the one for instantons in Eq. (27); condition (35) originates from Eq. (29) with the identification $z=x^{4}+i r$ and $r^{2}=\left(x^{1}\right)^{2}+\left(x^{2}\right)^{2}+\left(x^{3}\right)^{2}$.

\section{B. The ADHM construction for vortices}

From the fact that the $(N+k) \times k$ matrices $W$ and $\tilde{W}$ in Eq. (34) are analogous to the $2(N+k) \times 2 k$ matrix $V$ in Eq. (27) for the ADHM construction of instantons, the solutions can be recast into the $\mathrm{ADHM}$ form. In fact, for a given $\mathrm{ADHM}$ date $(T, \psi)$, the matrices $W$ and $\tilde{W}$ are solution of the "Dirac equations"

$$
\nabla_{v}^{\dagger} W=0, \quad \tilde{\nabla}_{v}^{\dagger} \tilde{W}=0
$$

where the "Dirac operators" for the vortices are given by

$$
\nabla_{v}^{\dagger} \equiv(\psi T-\bar{z}), \quad \tilde{\nabla}_{v}^{\dagger} \equiv(\psi T-z) .
$$

These Dirac operators are analogous to those of instantons in Eq. (26). Condition (35) is equivalent to the orthogonality conditions for matrices $W$ and $\tilde{W}$ :

$$
W^{\dagger} W=\mathbf{1}_{N}, \quad \tilde{W}^{\dagger} \tilde{W}=\mathbf{1}_{N}
$$

These conditions are also counterparts of Eq. (28) for instantons.

We need to check the existence of the inverse of $\Delta^{\dagger} \Delta$ in the ADHM construction for instantons, and therefore there should be a corresponding condition for the operators in Eq. (37) for vortices. However we will not study it in more detail in this paper and leave it as a future problem.

\section{Moduli space}

Here, we discuss the moduli parameters encoded in solutions (33), (34), and (35) and identify the moduli space. The solutions have the following redundancy in the moduli data $(T, \psi)$ :

$$
T \rightarrow U T U^{-1}, \quad \psi \rightarrow U \psi, \quad U \in U(k)
$$


They can be fixed as

$$
T=\left(\begin{array}{cccc}
t_{1} & & & \\
& t_{2} & & \\
& & \ddots & \\
& & & \\
& & & t_{k}
\end{array}\right), \quad \psi=\left(\begin{array}{cccc}
\rho_{11} & \rho_{12} & \cdots & \rho_{1 N} \\
\rho_{21} & \rho_{22} & \cdots & \rho_{2 N} \\
\vdots & & \ddots & \vdots \\
\rho_{k 1} & \rho_{k 2} & \cdots & \rho_{k N}
\end{array}\right), \quad t_{i} \in \mathbb{R}, \quad \rho_{i j} \in \mathbb{C} .
$$

The remaining $U(1)^{k}$ redundancy, $\rho_{i j} \rightarrow \exp \left(i \theta_{i}\right) \rho_{i j}$, can be fixed as $\rho_{i i} \in \mathbb{R}$. Therefore, the dimension of the $S[U(N) \times U(N)]$ vortex moduli space is $1 / 4$ of that of the (framed) $S U(2 N)$ instanton moduli space:

$$
\operatorname{dim}_{\mathbb{R}} \mathcal{M}_{k, N}^{\mathrm{vortex}}=2 k N
$$

If we further divide the moduli space by the $S U(N)$ (global) gauge symmetry $\psi \rightarrow \psi g, g \in$ $S U(N)$, the dimension of the moduli space becomes

$$
\operatorname{dim}_{\mathbb{R}}\left[\mathcal{M}_{k, N}^{\text {vortex }} / S U(N)\right]=\left\{\begin{array}{cc}
2 k N-N^{2}+1 & \text { for } k>N \\
k^{2}+1 & \text { for } k \leq N
\end{array} .\right.
$$

To determine the physical meaning of the moduli parameters, let us calculate

$$
\begin{aligned}
\operatorname{det} H & \propto \operatorname{det}\left(\mathbf{1}_{N}+\psi^{\dagger}\left(z \mathbf{1}_{k}-T\right)^{-2} \psi\right) \\
& \propto \operatorname{det}\left(\mathbf{1}_{k}+\left(z \mathbf{1}_{k}-T\right)^{-2} \psi \psi^{\dagger}\right) \\
& \propto \operatorname{det}\left(\left(z \mathbf{1}_{k}-T\right)^{2}+\psi \psi^{\dagger}\right) .
\end{aligned}
$$

Now, let us define a $k \times k$ complex matrix $Z$ by

$$
Z \equiv T+i R
$$

with a $k \times k$ Hermitian matrix $R$ satisfying

$$
i[T, R]+\psi \psi^{\dagger}=R^{2}
$$

Hence, Eq. (43) can be rewritten as

$$
\operatorname{det} H \propto \operatorname{det}(z-Z)\left(z-Z^{\dagger}\right) \text {. }
$$

Since the unbroken gauge symmetry becomes larger inside the vortex cores, the zeros of $\operatorname{det} H$ can be interpreted as the vortex positions. Therefore, Eq. (46) implies that the 
eigenvalues of $Z$ are the vortex positions. It is interesting to see that Eq. (45) is a remnant of the D-term condition of a Kähler quotient construction of the vortex moduli space. We thus obtain

$$
\mathcal{M}_{k, N} \simeq\left\{(Z, \psi) \mid \frac{1}{2}\left[Z^{\dagger}, Z\right]+\psi \psi^{\dagger}=R^{2}\right\} / U(k),
$$

where the $U(k)$ action is $Z \rightarrow U Z U^{-1}, \psi \rightarrow U \psi$, and $R \rightarrow U R U^{-1}$, as in Eq. (39). This is analogous to the Kähler quotient for $U(N)$ vortices on flat space $\mathbb{C}$ [1]. The moduli $Z$ represent the vortex positions, and the moduli $\psi$ can be identified as the orientational moduli, which are $k$ copies of $\mathbb{C} P^{N-1}$ for the separated vortices. The difference between our hyperbolic case and the flat case is that the right hand side of the D-term condition is $R^{2}$ in our case while it is just $\left(4 \pi / g^{2}\right) \mathbf{1}_{k}$ for the flat case. Since the eigenvalues of $R$ are the vortex positions in the $r$ coordinate, our D-term condition can be interpreted as a result of a position-dependent gauge coupling, as can be inferred from Eqs. (22) and (3). Note that the Kähler quotient in Eq. (47) does not give the correct moduli space metric, as in the case of the flat space.

Although the $\mathbb{C} P^{N-1}$ orientational moduli for a single vortex can be absorbed by a global gauge transformation, the relative orientations change the physical quantities. Let us consider a coincident vortex configuration in the $N=k=2$ case. If we set the matrices $T$ and $\psi$ as

$$
T=\left(\begin{array}{cc}
a & 0 \\
0 & -a
\end{array}\right), \quad \psi=\left(\begin{array}{cc}
\sqrt{r_{0}^{2}+a^{2}} & 0 \\
2 a & \sqrt{r_{0}^{2}-3 a^{2}}
\end{array}\right), \quad a \in\left[0, \frac{r_{0}}{\sqrt{3}}\right],
$$

the matrix $R$ is solved as

$$
R=\left(\begin{array}{cc}
r_{0} & \frac{r_{0}+i a}{\sqrt{r_{0}^{2}+a^{2}}} a \\
\frac{r_{0}-i a}{\sqrt{r_{0}^{2}+a^{2}}} a & r_{0}
\end{array}\right) .
$$

In this setting, the matrix $Z=T+i R$ has the degenerate eigenvalue $i r_{0}$; hence, the two vortices are coincident. Since the vortex position is independent of $a, a$ parameterizes the internal orientation of the vortices. Indeed, we can see from Eq. (48) that $(T, \psi)$ reduce to two copies of the data for an Abelian vortex at $a=0$ while $(T, \psi)$ become identical to the data of two vortices in the Abelian case at $a=r_{0} / \sqrt{3}$. We can confirm that the parameter $a$ is physical by observing the trace of the magnetic flux $F_{z \bar{z}}=-\tilde{F}_{z \bar{z}}$.

$$
i \sigma^{-1} \operatorname{tr} F_{z \bar{z}}=8 r^{2}\left(a^{2}+r_{0}^{2}\right)\left[\frac{1}{\left\{|z|^{2}+r_{0}^{2}+a(z-\bar{z})\right\}^{2}}+\frac{1}{\left\{|z|^{2}+r_{0}^{2}-a(z-\bar{z})\right\}^{2}}\right] .
$$




\section{SUMMARY AND DISCUSSION}

In summary, we have constructed a complete set of all the exact solutions of non-Abelian vortices in the $S[U(N) \times U(N)]$ gauge theory with a bi-fundamental scalar field on a hyperbolic plane with a certain curvature, by using $S O(3)$-invariant $S U(2 N)$ Yang-Mills instanton solutions. We also have established the ADHM construction for non-Abelian vortices. We further identified the complete moduli space of $k$ vortices, consisting of the moduli parameters encoded in the $k \times k$ matrix $Z$ for the position moduli and the $k \times N$ matrix $\psi$ for the orientational moduli. We have found the Kähler quotient for the moduli space, whose complex dimension is $k N$ as in the flat case.

Future works on related topics will include studies on the index theorem of vortices in quiver gauge theories, the explicit moduli space metric, and low-energy dynamics of vortices;

and an extension to arbitrary gauge groups [29], particularly $S O(N)$ and $U S p(2 N)$ [30], from the Yang-Mills instantons with corresponding groups. Since the hyperbolic surface is topologically equivalent to the flat space, quantum dynamics such as the vortex counting should be studied on the hyperbolic surface. In the case of $N=1$, our model reduces to the Abelian-Higgs model [23], in which the vortex equation is reduced to the Liouville equation. This implies the presence of a non-Abelian generalization of the Liouville equation.

\section{Acknowledgments}

This work is supported in part by Grant-in-Aid for Scientific Research, Nos. 23740226 (M.E.) and 20740141 (M.N.), from the Ministry of Education, Culture, Sports, Science and Technology (MEXT) of Japan, by the "Topological Quantum Phenomena" Grant-in Aid for Scientific Research on Innovative Areas, No. 23103515 (M.N.), and by Japan Society for the Promotion of Science (JSPS), Academy of Sciences of the Czech Republic (ASCR) under the Japan - Czech Republic Research Cooperative Program (M.E.).

\section{Appendix A: Hyperbolic plane}

The hyperbolic plane is a subspace in $\mathbb{R}^{2,1}$ given by

$$
X_{1}^{2}+X_{2}^{2}-X_{3}^{2}=-R^{2}
$$


The solution is parameterized by $\phi \in[0,2 \pi), \rho \in \mathbb{R}_{\geq 0}$ as

$$
X_{1}=R \cos \phi \sinh \rho, \quad X_{2}=R \sin \phi \sinh \rho, \quad X_{3}=R \cosh \rho .
$$

The metric is given by

$$
d s^{2}=d X_{1}^{2}+d X_{2}^{2}-d X_{3}^{2}=R^{2}\left(d \rho^{2}+\sinh ^{2} \rho d \phi^{2}\right) .
$$

which gives a constant scalar curvature $-1 / R^{2}$. The hyperbolic plane can be parametrized by a complex coordinate $y$ in an unit disc as

$$
y=\tanh \frac{\rho}{2} e^{i \phi}, \quad|y|<1
$$

Then, the metric becomes

$$
d s^{2}=2 g_{y \bar{y}} d y d \bar{y}=\frac{4 R^{2}}{\left(1-\left|y^{2}\right|\right)^{2}} d y d \bar{y} .
$$

An upper-half plane is also used to parameterize the hyperbolic plane, where a complex coordinate $z$ is given by

$$
z=\frac{y+i}{1+i y}, \quad \operatorname{Im} z>0
$$

In terms of this coordinate the metric becomes

$$
d s^{2}=R^{2} \frac{d z d \bar{z}}{(\operatorname{Im} z)^{2}}
$$

The $S O(2,1)$ isometry of $\mathbb{R}^{2,1}$ acts on $z$ as

$$
z \rightarrow \frac{a z+b}{c z+d}, \quad a, b, c, d \in \mathbb{R}
$$

\section{Appendix B: $S O(3)$-invariant ADHM data}

In this section, we show that the most general $S O(3)$-invariant ADHM data takes the form of Eq. (23). First, let us rewrite the pair of the matrices $\left(I, J^{\dagger}\right)$ as

$$
\left(\begin{array}{c}
I \\
J^{\dagger}
\end{array}\right)=\left(\begin{array}{c|c}
\phi_{4}+i \phi_{3} & i \phi_{1}+\phi_{2} \\
\hline i \phi_{1}-\phi_{2} & \phi_{4}-i \phi_{3}
\end{array}\right)=\phi_{i} \otimes \sigma_{i}+\phi_{4} \otimes \mathbf{1}_{2},
$$

where $\phi_{i}(i=1,2,3)$ and $\phi_{4}$ are arbitrary $k$-by- $N$ matrices. The $S O(3)$ transformations rotate $\phi_{i}$ and $\phi_{4}$ as

$$
\phi_{i} \rightarrow R_{i}^{j} \phi_{j}, \quad \phi_{4} \rightarrow \phi_{4}, \quad R^{T} R=\mathbf{1}_{3} .
$$


This transformation can be canceled if there exists a $U(k)$ gauge transformation such that

$$
U \phi_{i}=R_{i}^{j} \phi_{j}, \quad U \phi_{4}=\phi_{4}, \quad U \in U(k) .
$$

This implies that the matrix $U$ must be in a direct sum of several triplet and singlet representations: $\phi_{4}=0$ for the triplet part while $\phi_{i}=0$ for the singlet part. However, we can show that the triplet part does not satisfy the ADHM equations

$$
\frac{1}{2} \epsilon_{i j k}\left[T_{j}, T_{k}\right]+\left[T_{i}, T_{4}\right]+\frac{1}{2} \epsilon_{i j k}\left(\phi_{j} \phi_{k}^{\dagger}-\phi_{k} \phi_{j}^{\dagger}\right)+\phi_{i} \phi_{4}^{\dagger}-\phi_{4} \phi_{i}^{\dagger}=0 .
$$

For simplicity, let us consider the case of a single triplet representation $(k=3)$. The explicit form of the invariant data is given by

$$
\left(T_{i}\right)_{r s}=i a \epsilon_{i r s}, \quad\left(T_{4}\right)_{r s}=b \delta_{r s}, \quad\left(\phi_{i}\right)_{r A}=\delta_{r i} \psi_{A}, \quad \phi_{4}=0
$$

where $a, b \in \mathbb{R}$ and $\psi_{A} \in \mathbb{C}(A=1, \cdots, N)$ are arbitrary parameters. Then, Eq. (B4) reduces to

$$
\epsilon_{i r s}\left(a^{2}+\left|\psi_{A}\right|^{2}\right)=0
$$

This allows only a trivial solution $a=\psi_{A}=0$ for which the operator $\Delta^{\dagger} \Delta$ is not invertible at $x^{\mu}=(0,0,0, b)$. Therefore, there is no solution to the ADHM equations for the triplet representation. Similarly, we can in general show that there is no $S O(3)$-invariant ADHM data in a direct sum representation containg the triplet representation. Namely, the most general form of the $S O(3)$-invariant data should be in a direct sum of the singlet representations:

$$
T_{i}=\phi_{i}=0 \quad(i=1,2,3), \quad T_{4}=T, \quad \phi_{4}=\psi,
$$

where $T$ and $\psi$ are arbitrary $k$-by- $k$ and $k$-by- $N$ matrices, respectively.

[1] A. Hanany and D. Tong, "Vortices, instantons and branes," JHEP 0307, 037 (2003) hep-th/0306150].

[2] R. Auzzi, S. Bolognesi, J. Evslin, K. Konishi and A. Yung, "NonAbelian superconductors: Vortices and confinement in N=2 SQCD," Nucl. Phys. B 673, 187 (2003) hep-th/0307287.

[3] M. Eto, Y. Isozumi, M. Nitta, K. Ohashi and N. Sakai, "Moduli space of non-Abelian vortices," Phys. Rev. Lett. 96, 161601 (2006) hep-th/0511088; M. Eto, K. Konishi, G. Marmorini, 
M. Nitta, K. Ohashi, W. Vinci and N. Yokoi, "Non-Abelian Vortices of Higher Winding Numbers," Phys. Rev. D 74, 065021 (2006) hep-th/0607070.

[4] D. Tong, "TASI lectures on solitons: Instantons, monopoles, vortices and kinks," hep-th/0509216 D. Tong, "Quantum Vortex Strings: A Review," Annals Phys. 324, 30 (2009) arXiv:0809.5060 [hep-th]].

[5] M. Eto, Y. Isozumi, M. Nitta, K. Ohashi and N. Sakai, "Solitons in the Higgs phase: The Moduli matrix approach," J. Phys. A A 39, R315 (2006) hep-th/0602170.

[6] M. Shifman and A. Yung, "Supersymmetric Solitons and How They Help Us Understand NonAbelian Gauge Theories," Rev. Mod. Phys. 79, 1139 (2007) [hep-th/0703267]; M. Shifman and A. Yung, "Supersymmetric solitons," Cambridge, UK: Cambridge Univ. Pr. (2009) 259 p

[7] A. A. Abrikosov, "On the Magnetic properties of superconductors of the second group," Sov. Phys. JETP 5, 1174 (1957) [Zh. Eksp. Teor. Fiz. 32, 1442 (1957)].

[8] H. B. Nielsen and P. Olesen, "Vortex Line Models for Dual Strings," Nucl. Phys. B 61, 45 (1973).

[9] A. P. Balachandran, S. Digal and T. Matsuura, "Semi-superfluid strings in high density QCD," Phys. Rev. D 73, 074009 (2006) hep-ph/0509276]; E. Nakano, M. Nitta and T. Matsuura, "Non-Abelian strings in high density QCD: Zero modes and interactions," Phys. Rev. D 78, 045002 (2008) arXiv:0708.4096 [hep-ph]]; M. Eto and M. Nitta, "Color Magnetic Flux Tubes in Dense QCD," Phys. Rev. D 80, 125007 (2009) [arXiv:0907.1278 [hep-ph]]; M. Eto, E. Nakano and M. Nitta, "Effective world-sheet theory of color magnetic flux tubes in dense QCD," Phys. Rev. D 80, 125011 (2009) [arXiv:0908.4470 [hep-ph]].

[10] E. B. Bogomolny, "Stability of Classical Solutions," Sov. J. Nucl. Phys. 24, 449 (1976) [Yad. Fiz. 24, 861 (1976)]; M. K. Prasad and C. M. Sommerfield, "An Exact Classical Solution for the 't Hooft Monopole and the Julia-Zee Dyon," Phys. Rev. Lett. 35, 760 (1975).

[11] M. Shifman and A. Yung, "NonAbelian string junctions as confined monopoles," Phys. Rev. D 70, 045004 (2004) hep-th/0403149]; A. Hanany and D. Tong, "Vortex strings and fourdimensional gauge dynamics," JHEP 0404, 066 (2004) hep-th/0403158.

[12] N. Dorey, "The BPS spectra of two-dimensional supersymmetric gauge theories with twisted mass terms," JHEP 9811, 005 (1998) hep-th/9806056; N. Dorey, T. J. Hollowood and D. Tong, "The BPS spectra of gauge theories in two-dimensions and four-dimensions," JHEP 9905, 006 (1999) hep-th/9902134. 
[13] A. A. Belavin, A. M. Polyakov, A. S. Schwartz and Y. .S. Tyupkin, "Pseudoparticle Solutions of the Yang-Mills Equations," Phys. Lett. B 59, 85 (1975).

[14] S. Shadchin, "On F-term contribution to effective action," JHEP 0708, 052 (2007) hep-th/0611278]; T. Dimofte, S. Gukov and L. Hollands, "Vortex Counting and Lagrangian 3-manifolds," Lett. Math. Phys. 98, 225 (2011) [arXiv:1006.0977 [hep-th]]; Y. Yoshida, "Localization of Vortex Partition Functions in $\mathcal{N}=(2,2)$ Super Yang-Mills theory," arXiv:1101.0872 [hep-th]; G. Bonelli, A. Tanzini and J. Zhao, "Vertices, Vortices and Interacting Surface Operators," arXiv:1102.0184 [hep-th]; G. Bonelli, A. Tanzini and J. Zhao, "The Liouville side of the Vortex," JHEP 1109, 096 (2011) arXiv:1107.2787 [hep-th]]; A. Miyake, K. Ohta and N. Sakai, "Volume of Moduli Space of Vortex Equations and Localization," Prog. Theor. Phys. 126, 637 (2012) arXiv:1105.2087 [hep-th]]; T. Fujimori, T. Kimura, M. Nitta and K. Ohashi, "Vortex counting from field theory," JHEP 1206, 028 (2012) arXiv:1204.1968 [hep-th]].

[15] N. A. Nekrasov, "Seiberg-Witten prepotential from instanton counting," Adv. Theor. Math. Phys. 7, 831 (2004) hep-th/0206161.

[16] T. Inami, S. Minakami and M. Nitta, "Non-integrability of Self-dual Yang-Mills-Higgs System," Nucl. Phys. B 752, 391 (2006) hep-th/0605064.

[17] M. F. Atiyah, N. J. Hitchin, V. G. Drinfeld and Y. I. Manin, "Construction of Instantons," Phys. Lett. A 65, 185 (1978).

[18] M. Eto, Y. Isozumi, M. Nitta, K. Ohashi and N. Sakai, "Manifestly supersymmetric effective Lagrangians on BPS solitons," Phys. Rev. D 73, 125008 (2006) hep-th/0602289].

[19] T. Fujimori, G. Marmorini, M. Nitta, K. Ohashi and N. Sakai, "The Moduli Space Metric for Well-Separated Non-Abelian Vortices," Phys. Rev. D 82, 065005 (2010) arXiv:1002.4580 [hep-th]].

[20] M. Eto, T. Fujimori, M. Nitta, K. Ohashi and N. Sakai, "Dynamics of Non-Abelian Vortices," Phys. Rev. D 84, 125030 (2011) arXiv:1105.1547 [hep-th]].

[21] M. Eto, K. Hashimoto, G. Marmorini, M. Nitta, K. Ohashi and W. Vinci, "Universal Reconnection of Non-Abelian Cosmic Strings," Phys. Rev. Lett. 98, 091602 (2007) hep-th/0609214. M. Eto, L. Ferretti, K. Konishi, G. Marmorini, M. Nitta, K. Ohashi, W. Vinci and N. Yokoi, "Non-Abelian duality from vortex moduli: A Dual model of color-confinement," Nucl. Phys. B 780, 161 (2007) hep-th/0611313.

[22] M. Eto, T. Fujimori, S. Bjarke Gudnason, Y. Jiang, K. Konishi, M. Nitta and K. Ohashi, 
"Group Theory of Non-Abelian Vortices," JHEP 1011, 042 (2010) [arXiv:1009.4794 [hep-th]].

[23] E. Witten, "Some Exact Multi - Instanton Solutions of Classical Yang-Mills Theory," Phys. Rev. Lett. 38, 121 (1977).

[24] I. A. B. Strachan, "Low velocity scattering of vortices in a modified Abelian Higgs model," J. Math. Phys. 33, 102 (1992).

[25] A. D. Popov, "Integrability of Vortex Equations on Riemann Surfaces," Nucl. Phys. B 821, 452 (2009) [arXiv:0712.1756 [hep-th]]; A. D. Popov, "Non-Abelian Vortices on Riemann Surfaces: An Integrable Case," Lett. Math. Phys. 84, 139 (2008) arXiv:0801.0808 [hep-th]].

[26] S. Krusch and J. M. Speight, "Exact moduli space metrics for hyperbolic vortices," J. Math. Phys. 51, 022304 (2010) arXiv:0906.2007[hep-th]]; N. S. Manton and N. A. Rink, "Vortices on Hyperbolic Surfaces," J. Phys. A A 43, 434024 (2010) arXiv:0912.2058 [hep-th]]; P. Sutcliffe, "Hyperbolic vortices with large magnetic flux," arXiv:1204.0400 [hep-th].

[27] N. S. Manton and N. Sakai, "Maximally Non-Abelian Vortices from Self-dual Yang-Mills Fields," Phys. Lett. B 687, 395 (2010) arXiv:1001.5236 [hep-th]].

[28] A. D. Popov and R. J. Szabo, "Quiver gauge theory of nonAbelian vortices and noncommutative instantons in higher dimensions," J. Math. Phys. 47, 012306 (2006) hep-th/0504025; O. Lechtenfeld, A. D. Popov and R. J. Szabo, "Quiver Gauge Theory and Noncommutative Vortices," Prog. Theor. Phys. Suppl. 171, 258 (2007) [arXiv:0706.0979 [hep-th]].

[29] M. Eto, T. Fujimori, S. B. Gudnason, K. Konishi, M. Nitta, K. Ohashi and W. Vinci, "Constructing Non-Abelian Vortices with Arbitrary Gauge Groups," Phys. Lett. B 669, 98 (2008) arXiv:0802.1020 [hep-th]];

[30] M. Eto, T. Fujimori, S. B. Gudnason, K. Konishi, T. Nagashima, M. Nitta, K. Ohashi and W. Vinci, "Non-Abelian Vortices in $\mathrm{SO}(\mathrm{N})$ and USp(N) Gauge Theories," JHEP 0906, 004 (2009) arXiv:0903.4471 [hep-th]]; M. Eto, T. Fujimori, S. B. Gudnason, M. Nitta and K. Ohashi, "SO and US(p) Kahler and Hyper-Kahler Quotients and Lumps," Nucl. Phys. B 815, 495 (2009) [arXiv:0809.2014 [hep-th]]; M. Eto, T. Fujimori, S. B. Gudnason, Y. Jiang, K. Konishi, M. Nitta and K. Ohashi, "Vortices and Monopoles in Mass-deformed SO and USp Gauge Theories," JHEP 1112, 017 (2011) [arXiv:1108.6124 [hep-th]]. 